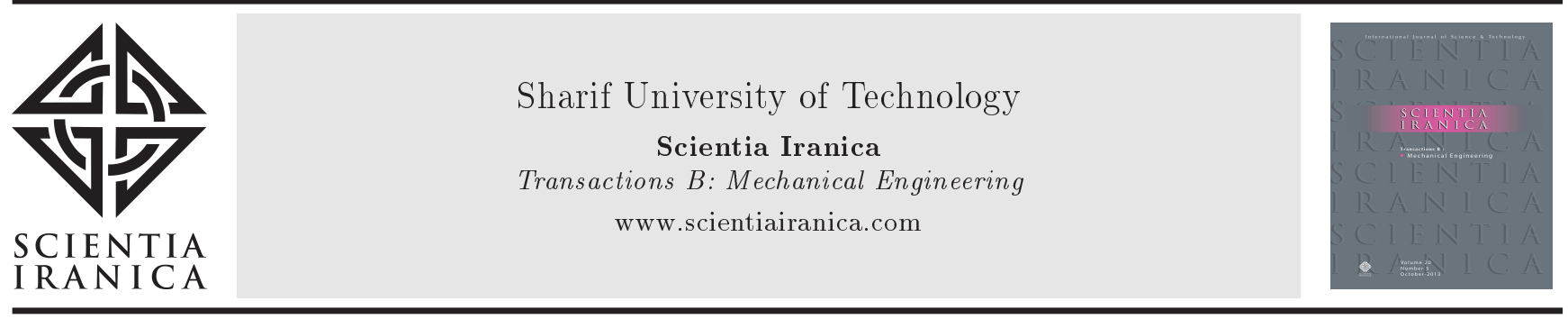

Research Note

\title{
A multi-step Gaussian filtering approach to reduce the effect of non-Gaussian distribution in aerial localization of an RF source in NLOS condition
}

\author{
S.M.M. Dehghan ${ }^{\mathrm{a}, \mathrm{b}}$ and H. Moradi ${ }^{\mathrm{a}, \mathrm{c}, *}$ \\ a. Advanced Robotics and Intelligent Systems Laboratory, School of Electrical and Computer Engineering, University of Tehran, \\ Teran, Iran. \\ b. Aerospace Research Complex, Malek-Ashtar University of Technology, Tehran, Iran. \\ c. Intelligent Systems Research Institute, SKKU, South Korea.
}

Received 14 May 2015; received in revised form 25 November 2015; accepted 10 May 2016

\author{
KEYWORDS \\ NLOS propagation; \\ Localization; \\ Particle filter; \\ Extended Kalman \\ filter; \\ Unscented Kalman \\ filter.
}

\begin{abstract}
The hybrid localization using Angle Of Arrival (AOA) and Differential Received Strength Signal Indicator (DRSSI) of an RF source with unknown power and NonLine-Of-Sight (NLOS) condition has been proven to be advantageous compared to using each method separately. In this paper, the initial hybrid method, which was implemented using particle filters due to the multi-modal/non-Gaussian nature of localization in NLOS condition, has been replaced by a multi-step Gaussian filtering approach which provides similar accuracy with better performance. This has been done using DRSSI input in the first step of the filtering to determine the linearization point, and then using AOA and DRSSI inputs together in the second step of the filtering to improve the localization accuracy. The proposed method has been implemented using Extended Kalman filter and Unscented Kalman filter. The simulation results show that the accuracy of the multi-step Gaussian filtering is comparable to the particle filtering approach with much lower computational load that is important for online localization of several RF sources. Furthermore, the effects of uncertainty on the propagation parameters have been studied to show that the robustness of the multi-step Gaussian filtering to the uncertainties is comparable to the particle filter approach.

(C) 2016 Sharif University of Technology. All rights reserved.
\end{abstract}

\section{Introduction}

Having Radio Frequency (RF) based communication devices is not a luxury anymore, and the majority of people carry a cell phone that uses RF signals for communication. Consequently, such devices can be used for localization of their owner/user, especially in search and rescue tasks (Figure 1). For instance, the signal from the cell phone of a person who is lost on a terrain can be used to localize him/her, even when

\footnotetext{
*. Corresponding author.

E-mail address: moradih@ut.ac.ir (H. Moradi)
}

he or she is unaware. That is why the researchers and governments have a tendency to use cell phones in the search and rescue mission for localization [1,2].

It is important to mention that due to the characteristics of the RF signals and the accuracy needed for localization, different approaches for using the RF signals for localization should be considered for different cases such as collapsed buildings, urban environment, and wide open areas. Furthermore, it is important to distinguish between direct visibility of the source of signal, i.e. being Line-Of-Sight (LOS), and indirect visibility of the source of signal, i.e. being Non-LineOf-Sight (NLOS). The existence of local scattering and 


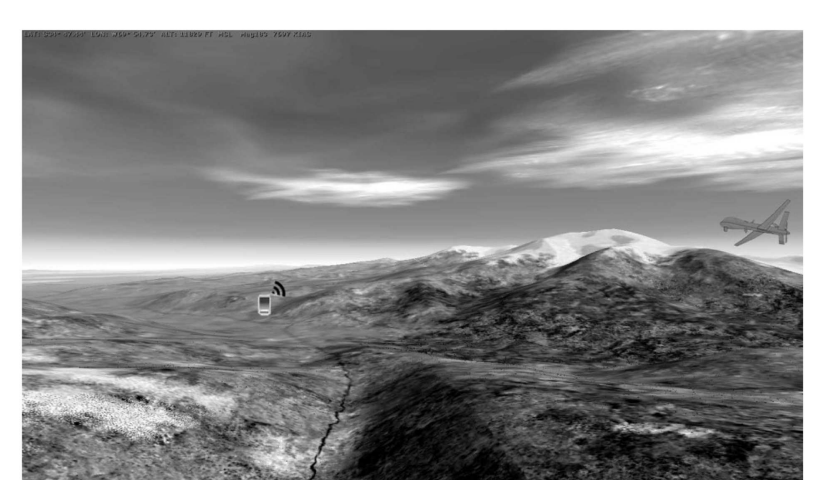

Figure 1. A UAV hovers over a terrain searching for an RF signal to localize the source (the cell phone is shown on the left and there is no LOS path between UAV and RF source).

shadowing/blockage in a NLOS situation makes it a much harder problem than LOS.

In practical sense, a UAV is flying over an open area such as a terrain (Figure 1) and tries to localize an RF source, which may also be called the target throughout the paper. In such a case, multipath and shadowing phenomena in NLOS propagation have different effects on AOA and RSSI measurements which can be used to reduce the localization error. Therefore, the authors developed a hybrid solution based on the RSSI and AOA approaches, named RSSI+AOA, to deal with NLOS propagation [3]. It is shown that when the transmitted power estimation is not required, it is possible to use DRSSI, instead of RSSI, and AOA together to provide desired accuracy with fewer numbers of particles, which reduces the processing time due to the reduction in the number of particles. DRSSI refers to the approach in which the difference between the received powers at two different way points is used to estimate the location of the RF source. It is noteworthy that it can achieve better accuracy than RSSI+AOA when they use the same number of particles. Another advantage of DRSSI+AOA, over $\mathrm{RSSI}+\mathrm{AOA}$, is that it can be used in cases in which the power of the target is time-varying and the use of multi-UAV for localization becomes important.

Due to the nonlinear nature of the pose estimation in NLOS propagation, the existence of non-modeled nonlinear dynamics in practical circumstances, and the multimodal nature of the problem, particle filter seemed more suitable than other options such as least square methods and Kalman filters. In other words, the effect of shadowing on the received signal has lognormal distribution that increases the non-linearity of the estimation. Furthermore, AOA propagation has a non-Gaussian term due to blockage and the problem is multimodal due to combined unknown transmitted power and unknown position of RF sources. Finally, particle filter seemed more robust against the uncertainties in the propagation parameters.
In this paper, the DRSSI+AOA approach is extended using multi-step Gaussian filtering to better eliminate the effects of nonlinear nature of NLOS localization. The proposed method has been implemented using extended Kalman filter and unscented Kalman filter and has been compared to the particle filtering based DRSSI+AOA. The results show that the performance of the proposed multi-step hybrid Gaussian filtering is comparable to the particle filtering based DRSSI+AOA with lower computational complexity. This has been done using DRSSI input in the first step of the filtering to determine the linearization point. Then, AOA and DRSSI inputs are used together in the second step of the filtering. The effect of uncertainty in propagation parameters using different filters is addressed in this paper to show that the robustness of multi-step Gaussian filtering can be comparable to particle filter. In other words, in this paper, we propose the multi-step Gaussian filtering which has the accuracy of particle filtering with lower computational complexity. It should be noted that this paper does not address the path planning of the flying robot and its way points are predetermined.

\section{Related work}

As mentioned in the introduction, researchers and governments have realized the capability in using $\mathrm{RF}$ signals for localization and started to investigate its possibility. For instance, Wireless Infrastructure over Satellite for Emergency Communications (WISECOM) aims to restore GSM (Global System for Mobile Communication) infrastructure over satellite and to use the restored system for tracking rescue teams and victims [1]. By this infrastructure, it is possible to provide information like the number of victims involved, their health condition, and how to reach them for rescue workers. I-LOV project [2] is another large scale project focused on setting up its own mobile phone network to provide emergency communication and localize the victims.

In the wide open areas, the use of UAVs to localize RF sources requires solving various issues such as cooperation [4], path planning [5-8], and optimal localization point [9-11] that ignited many studies in these fields. Localization of RF sources has been addressed using triangulation [12], least square methods [13], and Bayesian filters [12,14-16]. Lee et al. [14] used a dual-EKF algorithm to obtain the estimation of state values and unknown parameters of dynamic state model based on TDOA measured with two UAVs. Okello et al. [15] compared three tracking algorithms using a Gaussian Mixture Measurement Integrated Track Splitting Filter (GMM-ITSF), a multiple model filter with Unscented Kalman Filters (UKFs), and a multiple-model filter with Extended Kalman Filters 
(EKFs) for recursive estimation of emitter location using TDOA measurements formed by two UAVs. Kwon and Pack [16] extented out-of-order sigma-point Kalman filter (O3SPKF) method to handle mobile targets. The modified O3SPKF incorporates an adapted Sensor Fusion Quality (SFQ) principle to handle both static and mobile targets.

Localization of RF sources with unknown transmitted strength of signal is mostly performed in sensor networks, in which nodes are deployed densely and close to each other. To estimate the transmitted power in addition to the location of target, the maximum likelihood estimator [17-18] and/or a family of least square estimators [19] are used. In some cases, the path loss exponent is also estimated $[17,19]$. Unfortunately, the proposed approaches are limited to the given application, i.e. sensor networks, in which there are a large amount of measurements collected over time or by numerous stationary and close to each other sensors. On the other hand, the researchers tried to convert nonlinear and non-convex optimization problem of maximum likelihood estimator into a convex problem that can be solved efficiently [17]. There are also works to obtain analytical expressions for the Maximum Likelihood (ML) estimator [18] and achieve its global minimum [19]. Unfortunately, these approaches have high computational cost and heavy communication overhead that are not suitable for realtime localization. Furthermore, there is a limitation on uniquely estimating the unknown transmit power and location of a node [18]. In sensor networks, there are other protocol based power adaptive localization algorithms [20] which cannot be used in our desired application.

AOA-based localization is studied in many researches [21-23]. It has also been used in combination with Time of Arrival (TOA) or Time Difference of Arrival (TDOA) [24-25]. But, the differential RSSI (DRSSI) or RSSI ratio has rarely been used in sensor networks [26-28] or GSM-based localization [29]. To the best of our knowledge, DRSSI and its combination with AOA have not been used in aerial localization before.

Incorporating DRSSI observations with a low resolution AOA sensor resolves the sign ambiguity problem and decreases the dependency of DRSSI-based approach to its waypoints pairing rule [30]. Also, the fusion of these different sensors has been proposed to benefit from both DRSSI and AOA methods using their complementary performance in different propagation conditions. To benefit from the fusion of DRSSI and AOA, particle filter is the first choice due to the non-linearity of the estimation, non-Gaussian term in AOA propagation, non-modeled nonlinear dynamics in practical circumstances, and more robustness against the uncertainties in the propagation parameters [3].
However, particle filtering suffers from high computational complexity which linearly corresponds to the number of particles used. Consequently, in online localization of several RF-sources such as the mentioned application, decreasing the computational complexity becomes much important. That is why the multistep Gaussian filtering has been proposed to reduce the computational cost of localization by incorporating Kalman filtering in the process. It should be mentioned that in the proposed multi-step filtering, the nonGaussian component of AOA propagation is handled by estimating the location of the target using DRSSI, which has a Gaussian distribution. In the next step, the estimated location is used to limit the non-Gaussian term of AOA which allows the use of Kalman filtering.

\section{NLOS propagation}

Non-Line-Of-Sight (NLOS) is a term often used in radio communication to describe a radio channel or a link where there is no direct visual line between the transmitting and the receiving antennas. In this section, the basic concept of RF signal propagation in NLOS condition is explained. Propagation in NLOS condition is done by penetration, diffraction, reflection, and scattering mechanisms which are the causes for multipath and shadowing phenomena. Therefore, to simulate AOA and RSSI correctly in NLOS condition, it is important to use appropriate models for multipath and shadowing modeling.

The General path loss model [31], which is the most commonly used model for pass loss propagation in NLOS condition, is used in this study. Then, the effect of multipath and shadowing are added to model RSSI. In Figure 2, the effects of multipath and shadowing on the path loss are shown. Multipath is referred to receiving the reflection of transmitted signal from

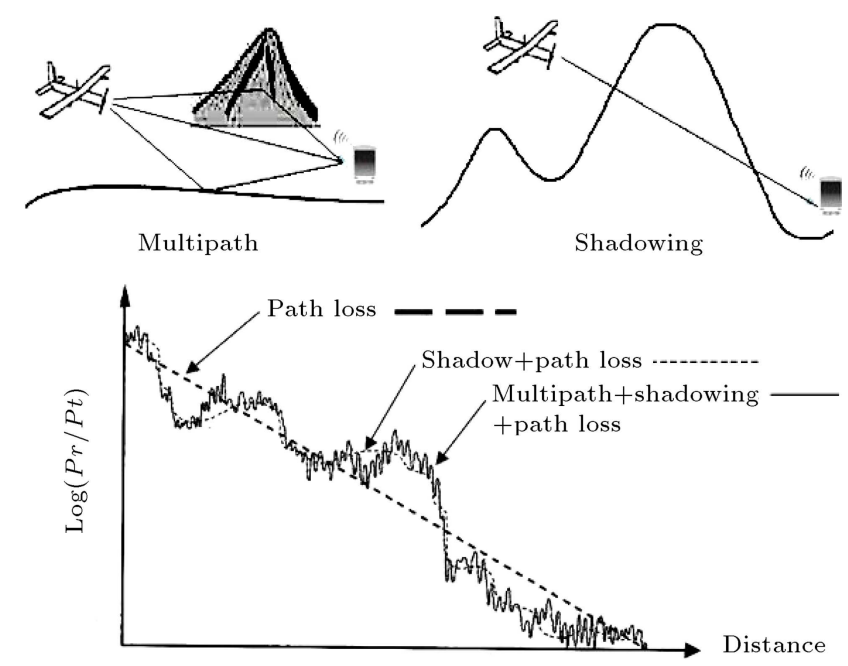

Figure 2. Signal attenuation due to multipath and shadowing (graph has been extracted from [31]). 


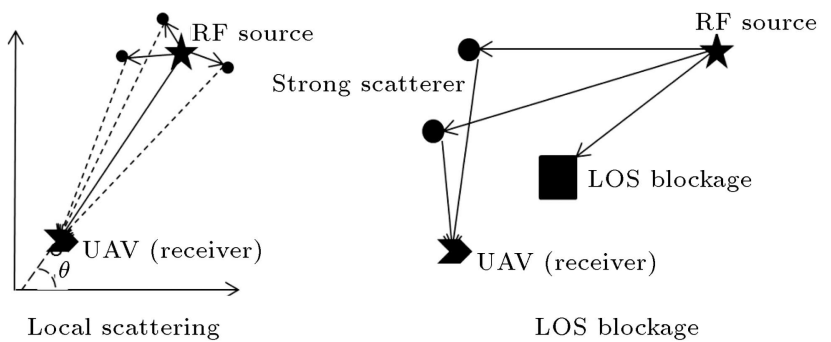

Figure 3. AOA propagation: (a) The local scatterers (dashed lines) while LOS condition holds; and (b) the strong scatterers when LOS blockage exists.

different directions and at different times that actually come from different paths. The effects of multipath on localization can be neglected assuming the possibility of averaging on the consecutive samples [29].

Signal attenuation due to shadowing has a lognormal distribution, which is equivalent to a normal distribution when it is expressed by dB (Figure 2). The Standard Deviation (SD) of shadowing effect $\left(\sigma_{S h}\right)$ is considered between $4.2 \mathrm{~dB}$ to $7.7 \mathrm{~dB}$ for suburb and $2.2 \mathrm{~dB}$ to $8.3 \mathrm{~dB}$ for urban [29].

To model AOA, the simplest and most appropriate model is presented in [32]. The basic components of this model are local scattering, probability of blockage, and a method for merging these (Figure 3 ). In other words, the effects of multipath and shadowing on the AOA measurements are modeled by these components.

In this model, spatial spreading and scattering in the vicinity of the source in LOS propagation scenarios is characterized by zero-mean Gaussian noise (Eq. (1)). Consequently, SD of local scattering $\left(\sigma_{L S}\right)$ is modeled, such that both spatial spreading and scattering are included.

$$
P_{L S}\left(\widehat{\theta} \mid X_{\mathrm{RF}}\right)= \begin{cases}\frac{1}{n} \frac{1}{\sqrt{2 \pi} \sigma_{L S}} e^{-\frac{\left(\hat{\theta}-\theta\left(X_{\mathrm{RF}}\right)\right)^{2}}{2 \sigma_{L S}^{2}}} & : \hat{\theta} \in\left[-\frac{\pi}{2}, \frac{\pi}{2}\right] \\ 0 & : \text { else }\end{cases}
$$

where $X_{\mathrm{RF}}$ is the location of the RF source, $\theta\left(X_{\mathrm{RF}}\right)$ is its computed AOA, and $\hat{\theta}$ is the observed AOA. AOAs are defined in the local coordinate of the AOA sensor mounted on the UAV in which $x$ axis is along the left wing of UAV and $y$ is along the longitudinal axis toward the nose of the UAV. $n$ is a normalization constant which is used to guarantee that the probability of the observation in the given limited range, i.e. $P_{L S}\left(\theta \mid X_{\mathrm{RF}}\right)$ in $-\frac{\pi}{2}$ and $\frac{\pi}{2}$ is one. It is computed once and used in all experiments. It should be mentioned that it is assumed that the AOA sensor only detects the signals in the $-\frac{\pi}{2}$ and $\frac{\pi}{2}$ region of its coordinate system, and any reflection from out of this range is ignored.

In NLOS propagation scenario, LOS blockage happens and AOA measurements are affected by strong scatterers, such as large far building or mountains, and are fairly uncorrelated. Consequently, AOA observations can be modeled using uniform distribution:

$$
P_{B l}\left(\hat{\theta} \mid X_{\mathrm{RF}}\right)= \begin{cases}\frac{1}{\pi}: & \hat{\theta} \in\left[-\frac{\pi}{2}, \frac{\pi}{2}\right] \\ 0: & \text { else }\end{cases}
$$

If the transmitter is narrowband and the receiver has small number of antennas, then the receiver can spatially resolve the AOA of the strongest arriving path. In this situation, AOA measurements are modeled based on a linear combination of Gaussian and blocked models (Eqs. (1) and (2)) using the probability of blockage, i.e. $\alpha_{B l}$ :

$$
\begin{gathered}
P_{\text {Narrow }}\left(\hat{\theta} \mid X_{\mathrm{RF}}\right)=\alpha_{B l} P_{B l}\left(\hat{\theta} \mid X_{\mathrm{RF}}\right)+\left(1-\alpha_{B l}\right) \\
P_{L S}\left(\hat{\theta} \mid X_{\mathrm{RF}}\right) .
\end{gathered}
$$

It should be mentioned that more complicated models are available which use the concept of scattering region as the locus of local scatterers [33]. However, the above model supports the main components of these complicated models and is sufficient in this study [32].

Finally, it is assumed that the RSSI and AOA noises are independent and their variances are time invariant. Furthermore, it is assumed that the RF source is stationary and does not move.

\section{Particle filter}

Due to the use of DRSSI+AOA approach, i.e. there is no need to estimate the power of the transmitter, the particles only represent the location of the RF source. On the other hand, due to the assumption that the navigation of the UAV is accurate considering its access to a precise integrated GPS/INS/Altimeter system and the relative accuracy needed for this problem, it can be assumed that the UAV is flying at a known altitude relative to the ground $\mathrm{RF}$ source. Consequently, the altitude of the RF source would not be needed to be estimated. Obviously, the approach can be extended considering the uncertainty in the UAV's pose. In other words, the state of each particle $i$ at time $t$ is the location of the source which is represented by $X_{\mathrm{RF}}^{[i]}(t)$. The detailed description of the steps of this filter was presented in [3]. In the following, only the PDF for updating particles' weights is presented for easier discussion. The new weights would be calculated by Eq. (4):

$$
\begin{aligned}
w^{[i]}(t)= & p\left(\hat{\theta}(t) \mid X_{\mathrm{RF}}^{[i]}(t)\right) * p\left(<P_{R x}(t-1)\right. \\
& \left.-P_{R x}(t)>\mid X_{\mathrm{RF}}^{[i]}(t)\right)
\end{aligned}
$$

in which $p\left(\hat{\theta}(t) \mid X_{\mathrm{RF}}^{[i]}(t)\right)$ is computed using Eq. (5): 


$$
\begin{gathered}
p\left(\hat{\theta}(t) \mid X_{\mathrm{RF}}^{[i]}(t)\right)=\frac{\alpha_{B l}}{\pi}+\left(1-\alpha_{B l}\right) \\
* \frac{1}{\sigma_{L S} \sqrt{2 \pi}} e^{\frac{-\left(\hat{\theta}(t)-\theta^{[i]}(t)\right)^{2}}{2 \sigma_{L S}^{2}}}
\end{gathered}
$$

and $p\left(<P_{R x}(t-1)-P_{R x}(t)>\mid X_{\mathrm{RF}}^{[i]}(t)\right)$ is computed using Eq. (6) [3]:

$$
\begin{aligned}
p\left(\mathrm{DPL}_{\mathrm{pwr}}(t) \mid \mathrm{DPL}_{\text {dist }}^{[i]}(t)\right) & =\frac{1}{2 \sqrt{\pi} \sigma_{s h}} \\
& e^{\frac{-\left(\mathrm{DPL}_{\mathrm{pwr}}(t)-\mathrm{DPL}_{\mathrm{dist}}^{[i]}(t)\right)^{2}}{4 \sigma_{s h} s^{2}}} .
\end{aligned}
$$

In these equations, $P_{R x}(t)$ is the received power at time $t, \mathrm{DPL}_{\mathrm{pwr}}(t)$ is the differential path loss according to the received power at time $t$ and $t-1, \mathrm{DPL}_{\text {dist }}^{[i]}(t)$ is the differential path loss according to the distance of particle $i$ from the robot's pose at time $t$ and its distance at time $t-1, \hat{\theta}(t)$ represents the measured angle of the target by the UAV at time $t$, and $\theta^{[i]}(t)$ represents the computed angle of particle $i$ with respect to the UAV location.

\section{Kalman filter design}

As mentioned before, shadowing and local scattering have Gaussian distribution which makes Gaussian filtering a suitable approach to handle their effects on localization. However, blockage has non-Gaussian characteristics and cannot be handled directly using Gaussian filtering. In other words, the localization accuracy of the Kalman filter which is affected by the blockage in AOA observations is strongly influenced by the probability of signal blockage.

\subsection{The system equation}

The location of the $\operatorname{UAV}\left(\mathbf{X}_{\mathrm{UAV}}=\left[\begin{array}{ll}x_{u} & y_{u}\end{array}\right]^{T}\right)$ is known and the location of the $\operatorname{RF}$ source $\left(\mathbf{X}_{\mathrm{RF}}=\left[\begin{array}{ll}x & y\end{array}\right]^{T}\right)$ is unknown and should be estimated. The differential RSSI is independent of the transmitted power, so state vector $\mathbf{s}(t)$ only includes the location of the RF source:

$$
\mathbf{s}(t)=\left[\begin{array}{l}
x(t) \\
y(t)
\end{array}\right] .
$$

\subsection{The motion model}

The location of the RF source is fixed on the ground, so the motion model of the UAV is used to predict the next state. Assuming the UAV's accurate motion, the motion model will be shown as in Eq. (8). In the equation, $t$ represents the current time frame and $t-1$ represents the previous time frame:

$$
\mathbf{s}(t)=\left[\begin{array}{l}
x(t) \\
y(t)
\end{array}\right]=\left[\begin{array}{ll}
1 & 0 \\
0 & 1
\end{array}\right]\left[\begin{array}{l}
x(t-1) \\
y(t-1)
\end{array}\right] .
$$

\subsection{Measurement update in the DRSSI approach}

Multi-step filtering uses the differential RSSI in the first step of the filtering to provide initial estimation of the state vector. So, a Gaussian filter based on DRSSI observation is developed in this section.

In this filter, the measurements are the difference of RSSI observations between two consecutive waypoints of the robot's path (Eq. (9)). In other words, the received power in the current time frame is compared or differentiated to/from the previous received power by the UAV.

$$
\begin{aligned}
& z=d \operatorname{Pr}(t)=\operatorname{Pr}(t)-\operatorname{Pr}(t-1), \\
& \operatorname{Pr}(t)=P t-P L(t) .
\end{aligned}
$$

Based on Eqs. (9) and (10), Eq. (11) can be extracted, which means that the difference of RSSI observations between two waypoints is equivalent to the difference of path loss in those waypoints:

$$
d P r(t)=-d P L(t) .
$$

Using the general loss model [31] (Eq. (12)), the observation equation, as a function of UAV's waypoint and the location of RF source, is derived (Eq. (13)) in which $P L_{d_{0}}$ means the path loss at the reference distance $d_{0}$ in $d B$ :

$$
\begin{gathered}
P L(t)=P L_{d_{0}}+10 \alpha_{P L} \log \\
\left(\frac{\left\|X_{\mathrm{RF}}-X_{\mathrm{UAV}}(t)\right\|}{d_{0}}\right) \\
z=h_{\mathrm{DRSSI}}\left(X_{\mathrm{RF}}, X_{\mathrm{UAV}}(t)\right)=-d P L(t)= \\
-10 \alpha_{P L} \log \frac{\sqrt{\left(x-x_{u}(t)\right)^{2}+\left(y-y_{u}(t)\right)}}{\sqrt{\left(x-x_{u}(t-1)\right)^{2}+\left(y-y_{u}(t)\right)}}
\end{gathered}
$$

\subsubsection{Linearization of the observation function}

Because of the nonlinearity of the observation function, an extended or an unscented Kalman filter should be used to estimate the state vector. To develop an extended Kalman filter, a linearized model by Jacobin is used:

$$
H(t)=\left.\frac{\partial h_{\mathrm{DRSSI}}}{\partial s}\right|_{s=\hat{s}(t-1)}=\left[\begin{array}{ll}
H_{1,1} & H_{1,2}
\end{array}\right],
$$

in which:

$$
\begin{aligned}
H_{1,1}= & \left.\frac{\partial h}{\partial x}\right|_{x=\hat{x}(t-1)}=\frac{-5 \alpha_{P L}}{\ln 10} \\
& * \frac{a\left(b^{2}+d^{2}\right)-b\left(a^{2}+c^{2}\right)}{\left(b^{2}+d^{2}\right)\left(a^{2}+c^{2}\right)},
\end{aligned}
$$




$$
\begin{aligned}
H_{1,2}= & \left.\frac{\partial h}{\partial y}\right|_{y=\hat{y}(t-1)}=\frac{-5 \alpha_{P L}}{\ln 10} \\
& * \frac{c\left(b^{2}+d^{2}\right)-d\left(a^{2}+c^{2}\right)}{\left(b^{2}+d^{2}\right)\left(a^{2}+c^{2}\right)} .
\end{aligned}
$$

In Eqs. (15) and (16) we have:

$$
\begin{aligned}
& a=\hat{x}(t-1)-x_{u}(t), \quad b=\hat{x}(t-1)-x_{u}(t-1), \\
& c=\hat{y}(t-1)-y_{u}(t), \quad d=\hat{y}(t-1)-y_{u}(t-1) .
\end{aligned}
$$

\subsubsection{The measurement noise}

The DRSSI measurement's noise variance is calculated based on the formula for calculating the difference between the two normal distributions:

$$
R=2 \sigma_{s h}^{2}
$$

\subsection{The measurement update in DRSSI+AOA based approach}

In this filter, the observations are DRSSI and AOA measurements:

$$
\begin{aligned}
Z= & {\left[\begin{array}{c}
\mathrm{DRSSI} \\
\mathrm{AOA}
\end{array}\right]=\left[\begin{array}{c}
h_{\mathrm{DRSSI}}\left(X_{\mathrm{RF}}, X_{\mathrm{UAV}}(t)\right) \\
h_{\mathrm{AOA}}\left(X_{\mathrm{RF}}, X_{\mathrm{UAV}}(t)\right)
\end{array}\right] } \\
& +\left[\begin{array}{l}
\delta_{1} \\
\delta_{2}
\end{array}\right] .
\end{aligned}
$$

The DRSSI observation equation, i.e., $h_{\mathrm{DRSSI}}\left(X_{\mathrm{RF}}\right.$, $X_{\mathrm{UAV}}(t)$ ), was presented in Eq. (13). The AOA observation equation, i.e. $h_{\mathrm{AOA}}\left(X_{\mathrm{RF}}, X_{\mathrm{UAV}}(t)\right)$, is as in Eq. (19):

$$
h_{\mathrm{AOA}}\left(X_{\mathrm{RF}}, X_{\mathrm{UAV}}(t)\right)=\tan ^{-1}\left(\frac{y-y_{u}(t)}{x-x_{u}(t)}\right) .
$$

5.4.1. The linearization of the observation function Developing the extended Kalman filter, the Jacobin is used to linearize the observation function:

$$
H(t)=\left.\frac{\partial Z}{\partial s}\right|_{s=\hat{s}(t-1)}=\left[\begin{array}{ll}
H_{1,1} & H_{1,2} \\
H_{2,1} & H_{2,2}
\end{array}\right],
$$

in which $H_{1,1}$ and $H_{1,2}$ are computed using Eqs. (15) and (16), respectively. The Jacobin of $h_{\mathrm{AOA}}\left(X_{\mathrm{RF}}\right.$, $\left.X_{\mathrm{UAV}}\right)$ is calculated using Eqs. (21) and (22):

$$
\begin{aligned}
H_{2,1} & =\left.\frac{\partial h_{\mathrm{AOA}}}{\partial x}\right|_{x=\hat{x}(t-1)} \\
& =-\left.\frac{\left(y-y_{u}(t)\right)}{\left(x-x_{u}(t)\right)^{2}+\left(y-y_{u}(t)\right)^{2}}\right|_{x=\hat{x}(t-1)}, \\
H_{2,2} & =\left.\frac{\partial h_{\mathrm{AOA}}}{\partial x}\right|_{y=\hat{y}(t-1)} \\
& =\left.\frac{\left(x-x_{u}[n]\right)}{\left(x-x_{u}(t)\right)^{2}+\left(y-y_{u}(t)\right)^{2}}\right|_{y=\hat{y}(t-1)} .
\end{aligned}
$$

\subsubsection{The measurement noise}

Due to AOA and DRSSI independent measurement noise assumption, the covariance of the measurements' noises is as in Eq. (23):

$$
R=\left[\begin{array}{cc}
2 \sigma_{s h}^{2} & 0 \\
0 & \sigma_{L S}^{2}
\end{array}\right]
$$

\subsection{The unscented Kalman filter}

Another approach to linearize a non-linear function is the Unscented Kalman Filter (UKF) that is used in the proposed hybrid DRSSI+AOA based localization approach. Similar to the previous approach, in which DRSSI is used to determine the predicted local scattering region and the linearization point, DRSSI is used to calculate the initial mean and sigma points in this approach. Sigma points, i.e. their values and weights, are chosen such that the mean and covariance of the distribution are modeled. Sigma points are passed through nonlinear function to recover the predicted mean and covariance. Based on the weighted average of the points, the new estimation and covariance are achieved. Finally, the observation, the predicted mean, and the predicted covariance are used to update the state estimation and its covariance.

One of the problems that occurs in the implementation of the UKF is that the covariance matrix should remain positive definite. However, this may not happen due to computational errors. Consequently, various decomposition methods of covariance matrix are used to overcome this problem. In this implementation, Cholesky decomposition is used. In this approach, instead of updating covariance matrix, the decomposition matrix is updated to avoid the mentioned problem.

It should be mentioned that, similar to the EKF approach, in this approach, a DRSSI-based UKF is developed using Eq. (13) and a DRSSI+AOA based UKF is developed using Eqs. (13) and (19). The motion model, the covariance of process noise, and the covariance of measurement noise are similar to EKF.

\subsection{The Gaussian approximation of $A O A$ measurements}

In EKF/UKF, the selection of the linearization point is crucial to properly model or approximate a nonGaussian process such as the localization in NLOS. Consequently, knowing the fact that the DRSSI observations always follow a Gaussian distribution, it can be used to determine the linearization point. In other words, DRSSI can provide the initial estimate of the target location. Then, the AOA measurements are included to improve the localization accuracy. This is why this approach is called a multi-step Gaussian filtering approach.

It is also important to mention that the blockage in NLOS propagation creates a uniform distribution in AOA measurements, while the local scattering creates 
a Gaussian distribution, whose mean was estimated in the first step of filtering. To be able to use Kalman filtering in the second step of the filtering, it is necessary to assume Gaussian distribution for the AOA observations. On the other hand, our experiments show that this assumption is fairly valid in the SD of local scattering and becomes invalid outside this region. Consequently, our Gaussian model of AOA measurements is only used in the SD of local scattering region around the angle of the estimated target position.

\section{Results}

Propagation simulation has been performed for a case in which the UAV is cruising at a fixed altitude above the terrains surrounding the RF source and a few kilometers away from it. In all simulations, the robot permanently cruises in circular path around the $\mathrm{RF}$ source and attempts to localize the RF source. The source can be anywhere within the circular path of the robot. The RSSI and AOA measurements can be performed at a distance less than a given threshold to the RF source. In this simulation, the maximum distance for measuring RSSI or AOA is set to $20 \mathrm{~km}$. Initially, it is assumed that a proper estimation of the characteristics of NLOS propagation and the environmental parameters, needed for path loss modeling, are known. They are chosen according to the characteristics of cell phones. This assumption is removed in the last test.

To simulate AOA sensors, it is needed to consider the available sensors in the market. Common Direction Finding (DF) sensors are simple energy detectors (e.g., spinning antennas) or phase sensitive systems (interferometer type) [34]. Small, commercial-off-the shelf (COTS) DF systems can provide a rough estimate of an emitter's bearing within a $90^{\circ}$ or $45^{\circ}$ sector [35]. In [36], a radio interferometric technique for determining bearings with an accuracy of approximately 3 degrees is described. Antenna arrays offer the possibility of high resolution angle estimates, but its price and weight limits the application. Particularly on UAVs, with hard payload restrictions, only small arrays can be used and array calibration techniques play a key role [34]. Consequently, to simulate a typical AOA sensor, a SD between six to ten degrees is used to simulate local scattering [37]. In total, six to twenty five degrees SD can cover the accuracy of all types of AOA sensor accuracies and environmental local scattering. It should be reminded that the SD value is used for Gaussian distribution to simulate the joint effect of the sensor accuracy and local scattering.

In the particle filter simulation, the number of particles is set to 400 . The developed Monte-Carlo simulations are based on the averages of 200 consec-

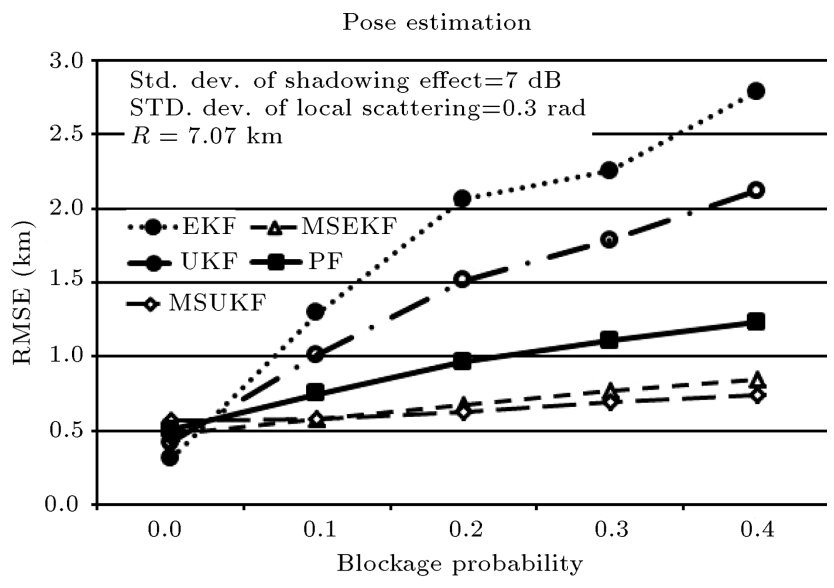

Figure 4. Comparison of filters in different blockage probability.

utive runs. This number will be increased gradually if the repeatability of the results mandates the increase in the number of runs.

To compare the different approaches, the Mean Square Error (MSE), which is composed of the bias and variance of the estimator (Eq. (24)), is used:

$$
\operatorname{MSE}(\hat{X})=\operatorname{Var}(\hat{X})+\operatorname{Bias}^{2}(\hat{X}) .
$$

\subsection{The effects of signal blockage}

In this test, the effect of signal blockage on the localization accuracy on developed filters has been compared to the particle filtering based DRSSI+AOA. The parameters are set as follows: the SD of local scattering is 0.3 radian, the $\mathrm{SD}$ of shadowing is $7 \mathrm{~dB}$, and the radius of the robot's path is $7.07 \mathrm{~km}$. Figure 4 shows that the accuracy of multi-step Gaussian filtering is better than particle filtering when there is blockage in the environment. It is interesting that the particle filter approach suffers more from blockage compared to the multi-step Gaussian filter when the number of particles is not increased. The reason is that the particle filter uses all of the blocked AOA observations which increase the error. In contrast, the multi-step Gaussian filtering focuses on the estimated location, which is estimated in the first step of the filtering using DRSSI observations, and eliminates any AOA observation which is out of the range. In other words, the accuracy of particle filtering can be increased by increasing the number of particles. However, it would increase the computational cost, while the multi-step Gaussian filtering would not face higher computational cost in such cases.

\subsection{The advantage of the DRSSI+AOA approach implemented using multi-step Gaussian and particle filters to the DRSSI approach in different blockage probability}

In the all studied filters, the benefit of using AOA observations in the localization is reduced when the 


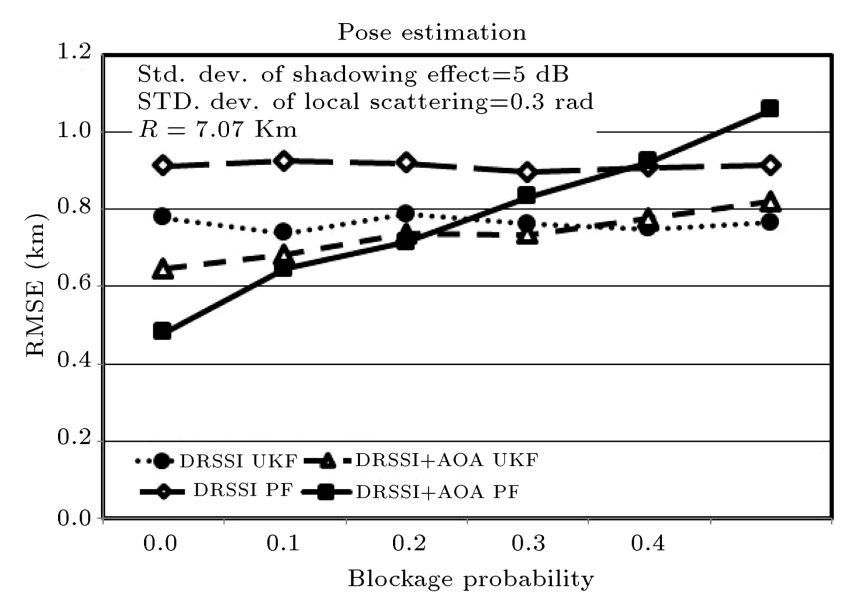

Figure 5. Comparison of the DRSSI and the DRSSI+AOA approaches implemented using the multi-step UKF and the particle filter in different blockage probability.

blockage probability increases. Thus, the DRSSI+AOA approach loses its advantage to the DRSSI in such cases. Although, this dependence on the blockage probability is less effective in the particle filter than the multi-step Gaussian filter (Figure 5), however, the benefit of using AOA observations is lost when the blockage probability passes 0.3 in the multi-step UKF approach and 0.4 in the particle filter approach. This means that the fusion of AOA with DRSSI in both approaches can increase the accuracy when the blockage probability is less than a threshold. It should be noted that the threshold for the particle filtering approach is lower than the threshold for the multi-step filtering, meaning that the particle filtering suffers more from blockage. It may be mentioned again that the particle filtering approach can be improved using higher number of particles that would increase the computational cost. It should also be noted that the multi-step EKF has similar performance to the multi-step UKF, and hence has not been illustrated to ease the presentation.

\subsection{Evaluation of the DRSSI+AOA approach implemented by the multi-step UKF, the multi-step EKF, and the particle filter in Radio Mobile propagation simulator}

In this simulation, the performance of the DRSSI+ AOA localization approach implemented by the multi- step UKF, the multi-step EKF, and the particle filter is evaluated using Radio Mobile propagation simulator. Radio Mobile is a computer simulation program used for predicting radio coverage of a base station, a repeater, or other radio networks. The simulator has been tested and the results have been validated with real air to ground communication data [38].

The main difference between the simulator used in Section 2 and the Radio Mobile simulator is that Radio Mobile uses the Longley-Rice algorithm to calculate the diffraction loss in addition to large scale fading. In other words, it explicitly considers the effects of obstacles in the simulation.

In this section, unlike the simulations implemented in the previous sections, the curvature of the earth is considered in the propagation simulation and localization. Also, the propagation simulation in Radio Mobile is done in the 3D space, i.e. the effect of height difference of UAV and RF source is considered in the amount of signal loss. Interestingly, the results show that the $2 \mathrm{D}$ simulation is accurate enough with negligible difference with the $3 \mathrm{D}$ simulation.

It should be mentioned that due to the lack of simulation of AOA propagation in Radio Mobile program, an integrated simulation including RSSI propagation in Radio Mobile and AOA propagation model described in Section 2 is used to evaluate the AOA-based approaches.

In Radio Mobile, the network parameters are set according to real values in GSM cellular network. The search area is set in a region near Tehran. The center of the region is at latitude of 34.81099 and longitude of 51.03735 and its radius is seven $\mathrm{km}$. The target is placed in two different locations, one on the center of the search area and the other one at latitude of 34.80014 and longitude of 51.02382. Propagation data are collected and then used off-line as input to the localization approaches.

The simulation consists of 16 waypoints with four turns around the search area. Table 1 shows the RMS of localization error in 500 consecutive runs for both specified points. In this simulation, location is chosen such that the terrain variation is comparable to the outcomes when the SD of shadowing is $9 \mathrm{~dB}$. According to the desired path, five out of 16 control points, are completely in blockage condition. Thus, the probability

Table 1. The RMS of localization error using Radio Mobile propagation simulator.

\begin{tabular}{|c|c|c|c|c|c|c|}
\hline \multirow[t]{2}{*}{$\begin{array}{c}\text { Localization } \\
\text { approach }\end{array}$} & \multicolumn{2}{|c|}{$\begin{array}{c}\text { RMSE (km) of } \\
\text { localization by particle } \\
\text { filter when the RF source is }\end{array}$} & \multicolumn{2}{|c|}{$\begin{array}{c}\text { RMSE (km) of } \\
\text { localization by multi-step } \\
\text { EKF when the RF source is }\end{array}$} & \multicolumn{2}{|c|}{$\begin{array}{c}\text { RMSE (km) of } \\
\text { localization by multi-step UKF } \\
\text { when the RF source is }\end{array}$} \\
\hline & $\begin{array}{l}\text { at the } \\
\text { center }\end{array}$ & $\begin{array}{l}\text { apart from } \\
\text { the center }\end{array}$ & $\begin{array}{l}\text { at the } \\
\text { center }\end{array}$ & $\begin{array}{l}\text { apart from } \\
\text { the center }\end{array}$ & $\begin{array}{l}\text { at the } \\
\text { center }\end{array}$ & $\begin{array}{l}\text { apart from } \\
\text { the center }\end{array}$ \\
\hline DRSSI+AOA & 1.6337 & 1.6764 & 1.2950 & 1.3437 & 1.4037 & 1.4598 \\
\hline
\end{tabular}


of blockage is set to 0.3 which is below the threshold discussed to benefit from AOA measurements. Also, the $\mathrm{SD}$ of scattering is selected 0.3 radians which is appropriate due to the selected terrain. The condition of the propagation is almost identical to both positions of the RF source. As it can be predicted, the multistep EKF and the multi-step UKF have more accuracy than the particle filter due the limited number of particles. This result is compatible with the results of the previous simulations.

It should be mentioned that the increase in the terrain variation would reduce the accuracy of the localization due to the introduction of more obstacles. Consequently, the complete resolution of this problem involves finding a solution to estimate the presence of the obstacles.

\subsection{The effects of unknown propagation parameters}

As mentioned, the propagation model is based on several parameters, i.e. the transmitter antenna's height, the path loss exponent, the SD of shadowing, the SD of local scattering, and the blockage probability, which are assumed known in the previous simulations. However, normally, there are uncertainties in the values of these parameters. Therefore, the robustness to the uncertainty of these parameters can be one of the criteria to select the suitable filter for the DRSSI+AOAbased localization approaches. In this section, the possible weakness of the proposed filters, for managing these uncertainties, is evaluated in comparison with the particle filter approach.

To study this robustness, the values of these parameters in propagation simulation are selected randomly in the possible ranges. The corresponding parameter values used in the filters are fixed based on selecting the best value over all simulation results.

The parameter changes are compared to a reference environment in which all the parameters are set at their mean. In each step of the simulation, only one of the parameters is changed randomly and other parameters have been kept fixed. In this reference environment, parameters are set as follows: The SD of local scattering is 0.3 radian, the SD of shadowing is $3.5 \mathrm{~dB}$, the probability of blockage is 0.3 , the path loss exponent is 3.5 , and the radius of robot's path is $7.07 \mathrm{~km}$. This Monte-Carlo simulation is based on the averages of 1000 consecutive runs. The RMSE of localization in the reference environment is shown in Table 2. In Table 3, the different values used to select the best SD value for the shadowing effect parameter are presented. The mean and maximum values of possible range of the SD of shadowing effect are used in the filters. Then, the RMSE of localization error is calculated based on the given fixed parameter values, used in the filters, and the unknown values are
Table 2. The RMSE of different filters in the reference propagation condition.

\begin{tabular}{cc}
\hline Filter & RMSE $\left(\widehat{\boldsymbol{P}_{\mathbf{R F}}}\right)$ \\
\hline Multi-step EKF & 0.8326 \\
Multi-step UKF & 0.5044 \\
PF & 0.8274 \\
\hline
\end{tabular}

Table 3. Selecting the best SD value for handling the shadowing effect of uncertainty.

\begin{tabular}{cccc}
\hline Filter & $\begin{array}{c}\text { Variation } \\
\text { range } \\
\text { of } \boldsymbol{\sigma}_{\boldsymbol{S} \boldsymbol{h}}\end{array}$ & $\begin{array}{c}\text { Selected } \\
\text { SD } \\
\text { for filter }\end{array}$ & $\begin{array}{c}\mathbf{R M S E} \\
\left(\widehat{\boldsymbol{X}_{\mathbf{R F}}}\right)\end{array}$ \\
\hline \multirow{2}{*}{ Multi-step EKF } & $0.1 \sim 7 \mathrm{~dB}$ & 7 & 0.6566 \\
& $0.1 \sim 7 \mathrm{~dB}$ & 3.5 & 2.2568 \\
Multi-step UKF & $0.1 \sim 7 \mathrm{~dB}$ & 7 & 0.6806 \\
& $0.1 \sim 7 \mathrm{~dB}$ & 3.5 & 1.6805 \\
& $0.1 \sim 7 \mathrm{~dB}$ & 7 & 1.2487 \\
Particle filter & $0.1 \sim 7 \mathrm{~dB}$ & 3.5 & 0.9227 \\
\hline
\end{tabular}

Table 4. The RMSE of different filters in various propagation conditions.

\begin{tabular}{ccccc}
\hline Filter & Par. & Range & $\begin{array}{c}\text { Best } \\
\text { SD in } \\
\text { filter }\end{array}$ & $\begin{array}{c}\text { RMSE } \\
\left(\widehat{\boldsymbol{X}_{\boldsymbol{R}}}\right)\end{array}$ \\
\hline \multirow{3}{*}{ Multi-step EKF } & $\sigma_{S h}$ & $0.1 \sim 0.5 \mathrm{rad}$ & $0.5 \mathrm{rad}$ & $0.7977 \mathrm{~km}$ \\
& $\alpha_{P L}$ & $3 \sim 4$ & 3.5 & $0.7751 \mathrm{dm}$ \\
& $\alpha_{B l}$ & $0.1 \sim 0.5$ & - & $0.8734 \mathrm{~km}$ \\
& & & & \\
Multi-step UKF & $\sigma_{L S}$ & $0.1 \sim 0.5 \mathrm{rad}$ & $0.5 \mathrm{rad}$ & $0.4792 \mathrm{~km}$ \\
& $\sigma_{P L}$ & $0.1 \sim 7 \mathrm{~dB}$ & $7 \mathrm{~dB}$ & $0.6908 \mathrm{~km}$ \\
& $\alpha_{B l}$ & $0.1 \sim 0.5$ & - & $0.6392 \mathrm{~km}$ \\
& & & 4 & $0.5191 \mathrm{~km}$ \\
& $\sigma_{L S}$ & $0.1 \sim 0.5 \mathrm{rad}$ & $0.5 \mathrm{rad}$ & $0.8709 \mathrm{~km}$ \\
& $\sigma_{S h}$ & $0.1 \sim 7 \mathrm{~dB}$ & $3.5 \mathrm{~dB}$ & $0.9072 \mathrm{~km}$ \\
& $\alpha_{P L}$ & $3 \sim 4$ & 3.5 & $0.7759 \mathrm{~km}$ \\
& $\alpha_{B l}$ & $0.1 \sim 0.5$ & 0.3 & $0.6861 \mathrm{~km}$ \\
\hline
\end{tabular}

randomly generated representing the actual values in the environment. The greater RMSE value represents greater sensitivity to the uncertainty of the parameter. Therefore, the best SD values, to be used in the filters, are selected based on the obtained smaller RMSE. As shown in Table 4, the best SD values for the multistep Gaussian filtering are the maximum values of the possible range, but the best SD value for the particle filter is the mean of the possible range.

Unknown Parameters that are studied in this test, their range of random variation, the selected 
value for use in the filters, and the resulting RMSE of localization are shown in Table 3 . Table 4 shows that the filters are almost robust against the given uncertainty in the propagation parameters. It should be noticed that using DRSSI observations makes the localization method independent to inaccurate selection of the path loss exponent and the SD of shadowing values, which is due to DRRSI's differential nature. This simulation shows that the robust advantage of the particle filter approach can also be provided by the multi-step Gaussian approach. This result needs more study in future researches to determine how much this advantage is caused by multi-step Gaussian and how much it is caused by the differential nature of the DRSSI observation.

\section{Conclusion}

In this paper, the particle filter-based DRSSI+AOA method has been replaced by a multi-step Gaussian filtering DRSSI+AOA approach. The proposed filter can eliminate the effects of nonlinear and nonGaussian nature of NLOS localization. As a result, the accuracy of DRSSI+AOA based localization using extended Kalman filter and unscented Kalman filter become comparable to the particle filter with much less computational load. This has been done using DRSSI input in the first step of the filtering to determine the linearization point, and then using AOA and DRSSI inputs together in the second step of the filtering to improve the localization accuracy. Finally, the robustness of these approaches to the uncertainties in the propagation parameters has been analyzed to show that the robustness of the multi-step Gaussian filtering can be comparable to the particle filter approach while it has lower computational cost. Furthermore, despite the comparable RMSE of localization by the proposed filters, i.e. EKF-based DRSSI+AOA and UKF-based DRSSI+AOA, to the particle filter, the maximum of estimation error is more in multi-step Gaussian filtering.

It should be mentioned that the proposed multistep Gaussian filtering approach can be used in any application with similar nature to the discussed application. In other words, if the fusion of two sources of data is done in which one source can be modeled using Gaussian distribution and the other one is nonGaussian, and then the first source can be used to determine the linearization point. Then, the linearization point is used to estimate the overall filtering using Gaussian assumption.

The future work can focus on the following areas. First, the generalization of this approach to similar problems can be investigated. Also, the restricted Gaussian modeling of the distribution performed in AOA propagation, which showed good results in multi- step Gaussian filtering, should be further investigated and generalized. Furthermore, the proposed approach would be tested in a real setup to confirm the validity of the simulation data. Finally, further analysis can be performed on selecting the propagation parameters to make the localization more robust and more accurate.

\section{References}

1. Berioli, M., Chaves, J.M., Courville, N., Boutry, P., Fondere, J., Skinnemoen, H., Tork, H., Werner, M. and Weinlich, M. "WISECOM: A rapidly deployable satellite backhauling system for emergency situations", International Journal of Satellite Communications and Networking, Special Issue on Emergency Telecommunications via Satellites, 29(5), pp. 419-440 (2011).

2. Zorn, S., Gardill, M., Rose, R., Goetz, A., Weigel, R. and Koelpin, A., A Smart Jamming System for UMTS/WCDMA Cellular Phone Networks for Search and Rescue Applications, 2012 IEEE MTT-S International Microwave Symposium Digest, pp. 1-3 (2012).

3. Dehghan, S.M.M., Farmani, M. and Moradi, H. "Aerial localization of an RF source in NLOS condition", IEEE International Conference on Robotics and Biomimetics, pp. 1146-1151 (2012).

4. Scerri, P., Glinton, R., Owens, S., Scerri, D. and Sycara, K., Geolocation of RF Emitters by Many $U A V s$, AIAA Infotech@Aerospace Conference, May (2007).

5. Dehghan, S.M.M., Moradi, H. and Shahidian, S.A.A. "Optimal path planning for DRSSI based localization of an RF source by multiple UAVs", Second RSI/ISM International Conference on Robotics and Mechatronics (ICRoM), pp. 558-563 (2014).

6. Dogancay, K. "Online optimization of receiver trajectories for scan-based emitter localization", IEEE Transactions on Aerospace and Electronic Systems, 43(3), pp. 1117-1125 (2007).

7. Dogancay, K. "UAV path planning for passive emitter localization", IEEE Transactions on Aerospace and Electronic Systems, 48(2), pp. 1150-1166 (2012).

8. Stachura, M. and Frew, E.W. "Cooperative target localization with a communication-aware unmanned aircraft system", AIA A Journal of Guidance, Control, and Dynamics, 34(5), pp. 1352-1362 (2011).

9. Bishop, A.N., Anderson, B.D.O., Fidan, B., Pathirana, P.N. and Mao, G. "Bearing-only localization using geometrically constrained optimization", IEEE Transactions on Aerospace and Electronic Systems, 45(1), pp. 308-320 (2009).

10. Drake, S., Brown, K., Fazackerley, J. and Finn, A. "Autonomous control of multiple UAVs for the passive location of radars", IEEE 5th International Conference on Intelligent Sensors, Sensor Networks, and Information Processing (ISSNIP'05), pp. 403-409 (2005). 
11. Purvis, K.B., Astrom, K.J. and Khammash, M. "Estimation and optimal configurations for localization using cooperative UAVs", IEEE Transactions on Control Systems Technology, 16(5), pp. 947-958 (2008).

12. Pack, D.J., DeLima, P., Toussaint, G.J. and York, G. "Cooperative control of UAVs for localization of intermittently emitting mobile targets", IEEE Transactions on Systems, Man, and Cybernetics Part B, 39(4), pp. 959-970 (August 2009).

13. Hmam, H. and Dogancay, K. "Passive localization of scanning emitters", IEEE Transaction on Aerospace and Electronics Systems, 46(2), pp. 944-951 (April 2010).

14. Lee, S.C., Lee, W.R. and You, K.H. "TDOA based UAV localization using Dual-EKF algorithm", International Journal of Control and Automation, 2(4), pp. 35-42 (December 2009).

15. Okello, N., Fletcher, F., Musicki, D. and Ristic, B. "Comparison of recursive algorithms for emitter localization using TDOA measurements from a pair of UAVs", IEEE Transactions on Aerospace and Electronic Systems, 47(3), pp. 1723-1732 (July 2011).

16. Kwon, H. and Pack, D.J. "A robust mobile target localization method for cooperative unmanned aerial vehicles using sensor fusion quality", Journal of Intelligent \& Robotic Systems, 65(1-4), pp. 479-493 (Jan. 2012).

17. Vaghefi, R.M., Gholami, M.R., Buehrer, R.M. and Ström, E.G. "Cooperative received signal strengthbased sensor localization with unknown transmit powers", IEEE Transactions on Signal Processing, 61(6), pp. 1389-1403 (March 2013).

18. Zafer, M., Ko, B.J. and Ho, I.W.H. "Transmit power estimation using spatially diverse measurements under wireless fading", IEEE/ACM Transactions on Networking, 18(4), pp. 1171-1180 (August 2010).

19. Wang, G., Chen, H., Li, Y. and Jin, M. "On receivedsignal-strength based localization with unknown transmit power and path loss exponent", IEEE Wireless Communications Letters, 1(5), pp. 536-539 (October 2012).

20. Ren, H. and Meng, M.Q.H. "Power adaptive localization algorithm for wireless sensor networks using particle filter", IEEE Transactions on Vehicular Technology, 58(5), pp. 2498-2508 (June 2009).

21. Eren, T. "Using angle of arrival (bearing) information for localization in robot networks", Turkish Journal of Electrical Engineering, 15(2), pp. 169-186 (2007).

22. Fidan, B., Drake, S.P., Anderson, B.D.O., Mao, G. and Kannan, A.A. "Collinearity problems in passive target localization using direction finding sensors", IEEE 5th International Conference on Intelligent Sensors, Sensor Networks and Information Processing (ISSNIP), pp. 115-120 (2009).

23. Li, M. and Lu, Y. "Angle-of-arrival estimation for localization and communication in wireless networks", 16th European Signal Processing Conference (EUSIPCO 2008), Lausanne, Switzerland (August 25-29 2008).

24. Mikhalev, A. and Ormondroyd, R.F. "Fusion of sensor data for source localization using the hough transform", IEEE 9th International Conference on Information Fusion (2006).

25. Du, H.J. and Lee, J.P.Y. "Simulation of multi-platform geolocation using a hybrid TDOA/AOA method", Technical Memorandum, Defence R\&D Canada Ottawa, TM 2004-256, December (2004).

26. Zhang, K.S., Xu, Y.M., Yang, W. and Zhou, Q. "Improved localization algorithm based on proportion of differential RSSI", Journal of Applied Mechanics and Materials, 192, pp. 401-405 (July 2012).

27. Kim, C.Y., Song, D., Xu, Y. and Yi, J. "Localization of multiple unknown transient radio sources using multiple paired mobile robots with limited sensing ranges", IEEE International Conference on Robotics and Automation (ICRA) (May 9-13 2011).

28. Wang, X. and Musicki, D. "Target tracking using energy based detections", IEEE 10th International Conference on Information Fusion (July 2007).

29. Dimitri, T., Fazli, E.H. and Werner, M. "A novel hybrid algorithm for passive localization of victims in emergency situations", International Journal of Satellite Communications and Networking, 29(5), pp. 461-478 (2011).

30. Dehghan, S.M.M., Shahidian, S.A.A. and Moradi, H. "Optimal pair selection in differential RSSI based localization of multiple RF sources", J. Dyn. Sys., Meas., Control, 137(11), pp. 114502-114506 (Aug 14, 2015). DOI: $10.1115 / 1.4031046$,

31. Jain, R., Channel Models a Tutorial In WiMAX forum AATG, pp. 1-6 (2007).

32. Ananthasubramaniam, B. and Madhow, U. "Cooperative localization using angle of arrival measurements in non-line-of-sight environments", ACM Workshop Mobile Entity Localization Tracking GPS-less Environments, pp. 117-122 (2008).

33. Le, K.N. "On angle-of-arrival and time-of-arrival statistics of geometric scattering channels", IEEE Transactions on Vehicular Technology, 58(8), pp. 4257-4264 (October 2009).

34. Passive Emitter Localization Using Airborne Bearing and TDOA, Fraunhofer Institute for Communication, Information Processing and Ergonomics, Sensor Data and Information Fusion Dept. Available: http://www.fkie.fraunhofer.de.

35. Walter, D., Klein, J., Kaupert, J., Bullmaster, C. and Chakravarthy, V. "Multiple UAV tomography based geolocation of RF emitters", SPIE, Defense Transformation and Net-Centric Systems, Proc., International Society for Optics and Photonics, 77070B.770708 (2010). 
36. Amundson, I., Sallai, J., Koutsoukos, X. and Ledeczi, A. "Mobile sensor waypoint navigation via RF-based angle of arrival localization", Hindawi Publishing Corporation, International Journal of Distributed Sensor Networks, Article ID 842107 (2012).

37. Ma, C. Techniques to Improve Ground-Based Wireless Location Performance Using a Cellular Telephone Network, PhD Thesis, Dept. of Geomatics Engineering, University of Calgary, Calgary, Canada (June 2003), Available: http://www. geomatics.ucalgary.ca/links/GradTheses.html

38. Analysis examples of Radio Mobile- RF propagatioon simulation software - high altitude balloon, http://radiomobile.pe1mew.nl/index.php?Analysis _examples: Ground_to_Air_propagation:High_altitude_balloon \#TOP

\section{Biographies}

Seyyed Mohammad Mehdi Dehghan received his $\mathrm{BSc}$ in Electrical and Computer Engineering, MSc in Artificial Intelligence and Robotics, and $\mathrm{PhD}$ in Control, respectively, in 1999, 2002, and 2015 from the University of Tehran. His research interests include autonomous aerospace systems, aerial robotics, navigation, UAVs cooperation, SLAM of RF sources, and attitude determination and control. He is now an Assistant Professor at the Aerospace Research Complex of the Malek-Ashtar University of Technology.

Hadi Moradi is an Associate Professor at the Electrical and Computer Engineering Department of the University of Tehran. He is also a member of Control and Intelligent Processing Center of Excellence. He received his $\mathrm{BSc}$ in Electrical Engineering from the University of Tehran and his MS and PhD degrees in Computer Engineering (Robotics), in 1994 and 1999, respectively from the University of Southern California. Before joining the Tehran University in 2008, he spent several years in industry as a research scientist. He joined USC at 2000 as a faculty member at the Computer Science Department. In 2004, he spent two years at the Intelligent Systems Research Center at Sungkyunkwan University, in South Korea till 2006. His current research interests include motion planning, manipulation, service robotics, educational robotics, and intelligent tutoring system, especially game-based teaching. 